\title{
Counterbalanced Valve Metal Oxide as a Reliable Dielectric Layer for Electrowetting-on-dielectric Devices
}

\author{
Supin Chen ${ }^{1 \dagger}$ and Chang-Jin "CJ" Kim ${ }^{1,2,3 *}$ \\ ${ }^{1}$ Bioengineering Department, University of California, Los Angeles (UCLA), \\ Los Angeles, CA 90095, U.S.A. \\ ${ }^{2}$ Mechanical and Aerospace Engineering Department, University of California, Los Angeles (UCLA), \\ Los Angeles, CA 90095, U.S.A. \\ ${ }^{3}$ California NanoSystems Institute (CNSI), University of California, Los Angeles (UCLA), \\ Los Angeles, CA 90095, U.S.A.
}

(Received May 29, 2019; accepted June 24, 2019)

Keywords: valve metal oxide, electrowetting, electrowetting-on-dielectric (EWOD), digital microfluidics

Valve metal oxide (anodized oxide) is used in a mirrored configuration to serve as selflimiting dielectric layers of electrowetting-on-dielectric (EWOD) devices. Resembling nonpolar electrolytic capacitors, the two opposing metal-dielectric layers always counterbalance each other so that voltages of any polarity can be applied without current flow. By using tantalum pentoxide as the valve metal oxide for experimental evaluation, the mirrored configuration is compared with the usual configuration and further compared with silicon oxide and silicon nitride, which are two dielectric materials commonly used for EWOD. Experiments with a range of applied biases confirm that in a mirrored configuration, one of the two metaldielectric-electrolyte combinations is always reverse-biased to prevent the current leakage, which is the most common mode of failure for the EWOD devices. The utility is demonstrated by manipulating droplets of $\mathrm{KCl}$ solution on both the parallel-plate and one-plate EWOD devices.

\section{Introduction}

\subsection{Dielectric layer for electrowetting}

Electrowetting is the apparent phenomenon of contact angle decrease, i.e., wetting, when an electric field is applied between a substrate electrode and an electrically conductive liquid on it, and can actuate the liquid for physical responses. ${ }^{(1-4)}$ To apply a higher voltage for a stronger actuation force without electric current between the liquid and the substrate, a dielectric layer was incorporated between them ${ }^{(5)}$ in a configuration named electrowettingon-dielectric (EWOD). ${ }^{(3)}$ By arranging electrodes side-by-side, electric potentials can be applied sequentially for basic liquid manipulations, such as droplet generation, movement, splitting, and merging. ${ }^{(6)}$ Because it enables control over the droplet shape and liquid flow using only electrical signals, EWOD actuation has found many applications, including low-

\footnotetext{
*Corresponding author: e-mail: cjkim@ucla.edu

${ }^{\dagger}$ Current address: Neuralink Corp., San Francisco, CA 94107, U.S.A.

https://doi.org/10.18494/SAM.2019.2449
} 
power displays, ${ }^{(1)}$ variable-focus lenses, ${ }^{(7)}$ thermal switches, ${ }^{(8)}$ electrical switches, ${ }^{(9)}$ energy harvesting, ${ }^{(10)}$ and rheometers. ${ }^{(11,12)}$ As a lab-on-a-chip platform, ${ }^{(13)}$ EWOD has been widely used for applications as diverse as combinatorial synthesis, ${ }^{(14)}$ sample processing ${ }^{(15,16)}$ enzymelinked immunosorbent assay (ELISA), ${ }^{(17)}$ and cell manipulation. ${ }^{(18)}$

Dielectric properties strongly affect the performance and reliability of EWOD devices. Desired would be a high dielectric constant and a high breakdown strength, so the dielectric could be deposited as a thin layer, which would reduce the voltage that needs to be applied for a given electrowetting effect. ${ }^{(19)}$ It should also be easily deposited as a pinhole-free layer for reliable performance with low fabrication cost. Many dielectrics have been used for EWOD or similar devices [e.g., photoresists, polydimethylsiloxane (PDMS), Parylene, silicon dioxide, silicon nitride, barium strontium titanite (BST), and bismuth zinc niobate (BZT)], ${ }^{(20,21)}$ but it has been difficult to achieve both high electrical performance and easy deposition, because one generally limits the other.

\subsection{Anodic oxide as the dielectric layer for EWOD}

Anodic oxides are oxides of valve metals, such as aluminum (Al), bismuth (Bi), tantalum (Ta), and antimony (Sb), and are electrochemically grown by the anodization or anodic oxidation of the metal. These have been extensively used as dielectrics in electrolytic capacitors for their large capacitance owing to their high dielectric constant and large surface area. ${ }^{(22)}$ Recently, these valve metals have been proposed as promising dielectric layers for EWOD owing to their high dielectric constants (8-110), high inherent breakdown strength, and simple low-cost fabrication (low deposition temperature and free of reactive gases). ${ }^{(23-25)}$ Furthermore, grown electrochemically with higher growth rates along weaker insulation paths, the formed layer is considered uniform with few pinholes. ${ }^{(26)}$ However, valve metal oxides are so named because of their current rectifying behavior, which can impose a restricted range of allowable voltages when used for EWOD. ${ }^{(25,27)}$ Leakage current (cathodic) is almost zero under the anodization bias, but the leakage current (anodic) can become significant under the opposite bias. ${ }^{(26)}$ The current rectifying behavior of the valve metal oxide used for EWOD was demonstrated by Huang et al., ${ }^{(27)}$ who showed that the devices with anodized $\mathrm{Ta}_{2} \mathrm{O}_{5}$ performed well when the droplet was applied with a reverse-biased DC or low-frequency voltages under $100 \mathrm{~Hz}$; however, the performance deteriorated severely in the case of forward-biased DC or high-frequency AC voltages.

Despite their current rectifying effect, progress has been made to reliably use valve metal oxides as the primary dielectric in EWOD devices. Huang et al. ${ }^{(27)}$ demonstrated that when $\mathrm{Ta}_{2} \mathrm{O}_{5}$ was sputter-deposited (instead of grown electrochemically) onto Ta and then anodized, the final dielectric layer was pinhole-free and insensitive to polarities when tested with $13 \mathrm{~V}(13 \%$ of the anodization formation potential) of actuation, which was sufficient for a contact angle reduction of $18^{\circ}$. It was not tested with higher voltages (greater than $50 \%$ of the anodization formation potential), under which a current rectifying effect would be more notable. ${ }^{(26)}$ There is also a concern with the utility of sputtering $\mathrm{Ta}_{2} \mathrm{O}_{5}$ before anodizing because an etch process to pattern electrodes would expose Ta sidewalls without the sputtered oxide. In other works, 
Dhindsa et al. ${ }^{(24)}$ demonstrated a reliable EWOD operation within ranges of voltage polarities and droplet compositions, so that any defects in the dielectric would be "self-healed" by further anodization as shown for electrolytic capacitors. ${ }^{(28)}$

The rectifying effect of the valve metal oxide was also utilized to create a polarity-dependent EWOD actuation for moving droplets across a surface without the sequential activation of electrodes. ${ }^{(29,30)}$ The authors used a single high-resistivity electrode and designed exposed aluminum vias through a thermal oxide dielectric, which were spaced so that at least two vias were always beneath the droplet. The native oxide that formed on aluminum (a valve metal) acted as a diode so that current flowed through a via on the low-voltage side but impeded by a via on the high-voltage side of the droplet. The resulting charge accumulation on the highvoltage side caused the droplet to move in its direction. Although current flowed through the droplet, it was limited by the use of diodes with opposite polarities.

\subsection{Anodic oxides of mirrored configuration for EWOD}

Although electrolytic capacitors use valve metal oxides that have current rectifying effects, nonpolar electrolytic capacitors can be formed when the anodized dielectric is incorporated into both capacitor electrodes in a mirrored (or symmetric) configuration. ${ }^{(31)}$ In this configuration, one of the two opposing valve metal oxide dielectrics will always be under the reverse bias to restrict current. Following the proposal to adopt the mirrored configuration of nonpolar electrolytic capacitors for the dielectric configuration of EWOD devices and the experimental confirmation using $\mathrm{Al}$ as the valve metal in a parallel-plate EWOD device, ${ }^{(32)}$ in the current report, we adopt Ta as a more suitable valve metal and implement the technology to not only the usual parallel-plate EWOD devices, as illustrated in Fig. 1, but also the coplanar EWOD devices. The valve metal oxides are utilized in such a way that always one is under the reverse bias to limit current. For parallel-plate EWOD devices, the mirrored configuration differs from the typical parallel-plate EWOD, which uses a thinner or no dielectric on one plate. After the basic EWOD characterization of $\mathrm{Ta}_{2} \mathrm{O}_{5}$ against silicon oxide and silicon nitride, the mirrored configuration is evaluated against the typical configuration with $\mathrm{Ta}_{2} \mathrm{O}_{5}$ as the dielectric. For coplanar EWOD devices, commonly used for single-plate open operations, the mirrored configuration does not differ from the typical. The performance is evaluated by performing the basic EWOD characterization and demonstrating droplet movement.

\section{Experimental Procedure}

\subsection{Anodization}

For anodization (or anodic oxidation), the sample valve metal electrode and a counter (unreactive) electrode are immersed in a conducting electrolyte solution, and a bias is applied between the two electrodes (Fig. 2). Oxidation occurs at the anode and reduction occurs at the cathode. ${ }^{(26)}$ The sample valve metal electrode is connected as the anode (positively charged), where the applied electric field causes oxide growth from both the metal/oxide interface via 


\section{Typical configuration}
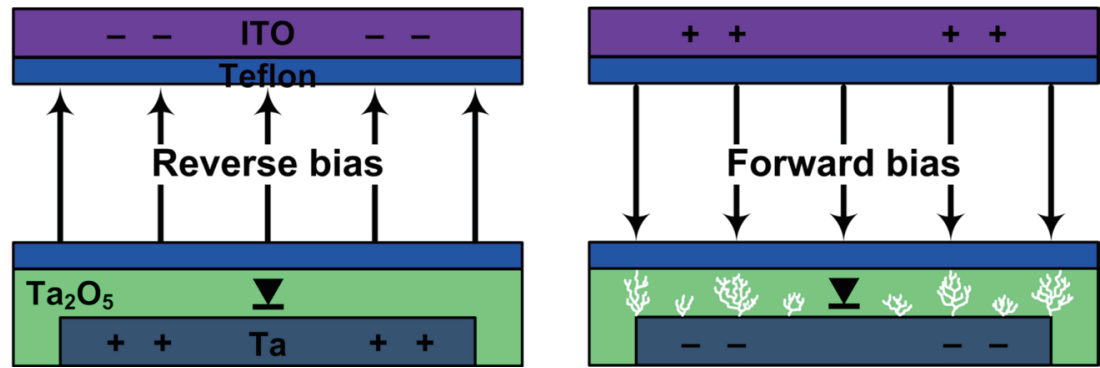

(a)

\section{Mirrored configuration}
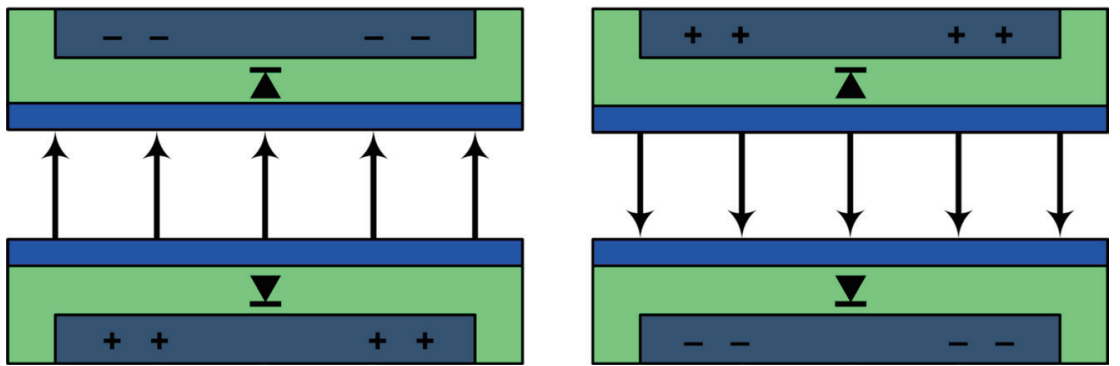

(b)

Fig. 1. (Color online) EWOD device with a valve metal oxide (e.g., $\mathrm{Ta}_{2} \mathrm{O}_{5}$ ) as the dielectric. (a) The existing configuration is limited by the rectifying behavior. Under reverse (negative) bias, the oxide layer (colored green) blocks current. However, under forward (positive) bias, the oxide layer passes current. Dielectric breakdown is indicated by a white dendrite pattern in the oxide. (b) A mirrored configuration is proposed to ensure that one of the opposing oxide layers is always under reverse bias (the correct bias for this paper) to restrict current and achieve a reliable device.

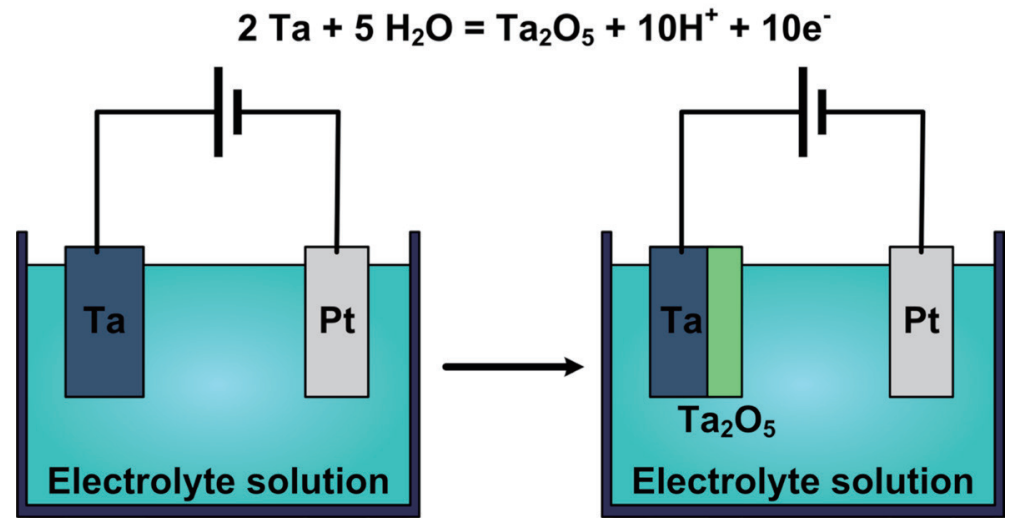

Fig. 2. (Color online) Anodization setup: platinum was used as a counter electrode and connected to the ground terminal of the power supply. The electrolyte solution was mixed during the process by a magnetic stirrer. 
the inward migration of oxygen and the oxide/electrolyte solution interface via the outward migration of metal. ${ }^{(33)}$ Modeled as a linear function of the applied voltage, ${ }^{(34)}$ the oxide thickness from anodization can be precisely controlled by adjusting the applied anodization voltage. However, the anodization constant (or proportionality constant of oxide thickness with respect to voltage) depends on the growth conditions (e.g., electrolyte, concentration, temperature, and $\mathrm{pH}){ }^{(26)}$

\subsection{Sample fabrication}

All of the samples were fabricated on clean glass slides ( $0.7 \mathrm{~mm}$ thick) (Fig. 3). For parallelplate EWOD tests, $500 \mathrm{~nm}$ of Ta was sputter-coated on the glass as the EWOD electrodes, on which $\mathrm{Ta}_{2} \mathrm{O}_{5}$, silicon nitride, or silicon oxide was formed as the dielectric. Ground plates were also prepared by evaporating 20/200 $\mathrm{nm}$ of chrome/gold as the reference electrode for a typical parallel-plate EWOD architecture. A tantalum pentoxide dielectric was grown from sputtered tantalum electrodes by anodization in a room-temperature $0.05 \mathrm{M}$ solution of citric acid in deionized water using a constant current step of $0.1 \mathrm{~mA} / \mathrm{cm}^{2}$. The constant current step was followed by a 1-h constant voltage step of $50 \mathrm{~V}$ (for 83-nm-thick $\mathrm{Ta}_{2} \mathrm{O}_{5}$ ) or $100 \mathrm{~V}$ (for 165 -nmthick $\mathrm{Ta}_{2} \mathrm{O}_{5}$ ). On other samples intended for comparison, silicon oxide and silicon nitride were deposited onto tantalum electrodes at a thickness of $165 \mathrm{~nm}$ using PECVD. No dielectric was deposited on the gold-coated ground plates.

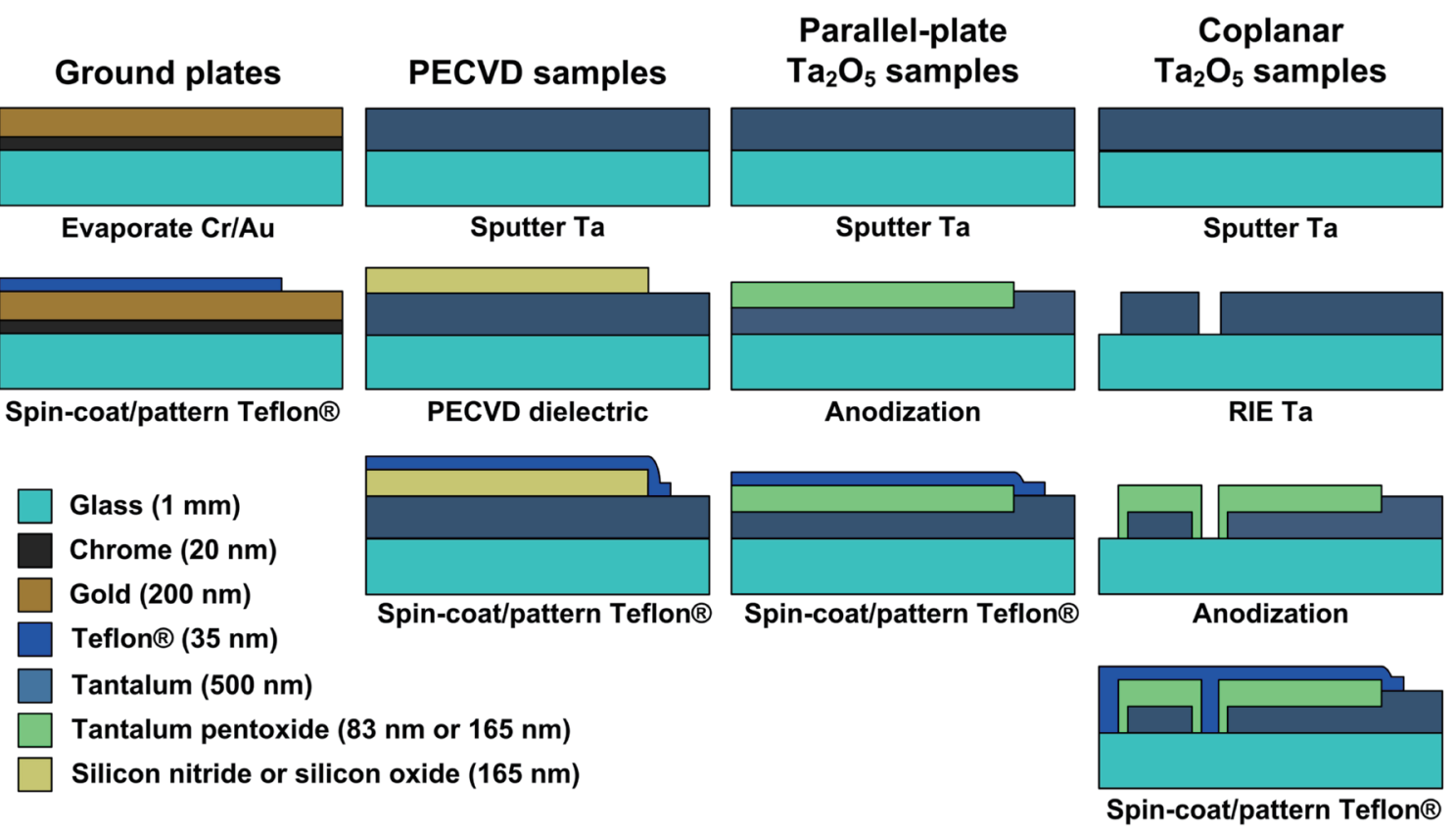

Fig. 3. (Color online) Process flow to fabricate EWOD samples to test and compare a mirrored configuration of valve metal oxide $\left(\mathrm{Ta}_{2} \mathrm{O}_{5}\right.$ shown) as an EWOD dielectric. 
For coplanar EWOD tests, two different sets of electrodes were prepared. The first was to actuate sessile droplets to measure the contact angle changes similarly to that described by $\mathrm{Yi}$ and $\mathrm{Kim},{ }^{(35)}$ and the second consisted of two rows of square electrodes to move droplets side-to-side with the sequential activation of neighboring electrodes (Fig. 4). The coplanar EWOD device fabrication began with $500 \mathrm{~nm}$ of sputtered tantalum on glass slides. The electrodes were patterned by photolithography with an AZ 4620 photoresist ( $6.2 \mu \mathrm{m}$ thick, MicroChemicals GmbH, Germany) and dry-etched by RIE (SF 6 gas, 40 mTorr, 35 V DC bias, and $115 \mathrm{~W}$ forward RF power). Before anodization, the photoresist was stripped using a solvent wash and $\mathrm{H}_{2} \mathrm{SO}_{4}: \mathrm{H}_{2} \mathrm{O}_{2}$ (4:1) cleaning step. Tantalum pentoxide was anodized under the same conditions as the parallel-plate samples. A hydrophobic layer of Teflon AF $2400^{\circledR}(35 \mathrm{~nm})$ was spin-coated onto all of the samples and patterned by lift-off to expose the bare metal for electrical contact.

\subsection{Current leakage and contact angle measurements}

In the typical parallel-plate EWOD configuration, a dielectric is used on the main plate with actuation electrodes but not on the ground plate with a reference electrode other than

Mask layout
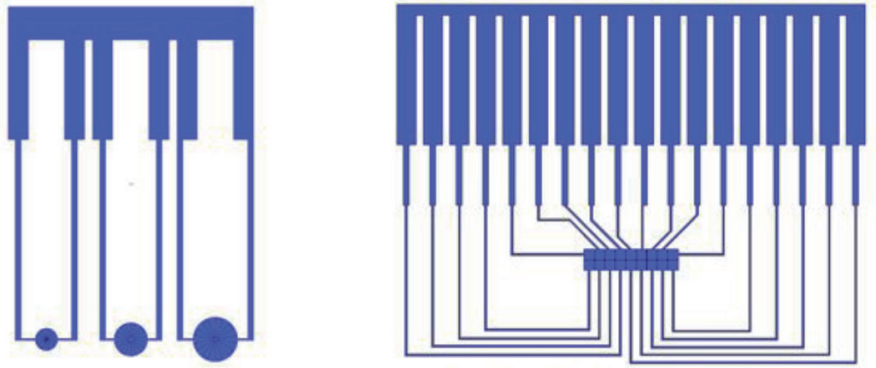

Zoomed in on
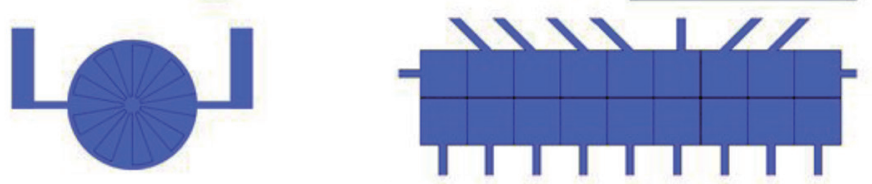

EWOD electrodes

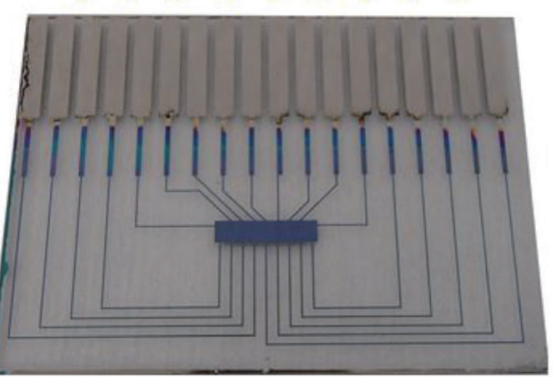

(a)

(b)

Fig. 4. (Color online) Coplanar EWOD devices with $\mathrm{Ta}_{2} \mathrm{O}_{5}$ (anodized Ta) as the dielectric. (a) Sessile droplet actuation design, adapted from Yi and Kim, ${ }^{(35)}$ to measure the contact angle change. (b) Two rows of square electrodes (1 $\times 1 \mathrm{~mm}^{2}$ ) for moving droplets side-to-side. 
the hydrophobic topcoat. In the mirrored configuration for parallel-plate EWOD, both plates include a dielectric $\left(\mathrm{Ta}_{2} \mathrm{O}_{5}\right.$ was used for testing). In the mirrored configuration for coplanar EWOD, $\mathrm{Ta}_{2} \mathrm{O}_{5}$ insulated both actuation and reference electrodes on a single plate.

Test droplets consisted of a 1:1 mixture of glycerin and $\mathrm{KCl}$ in water standard solution in order to prevent evaporation (with glycerin) and ensure conductivity (with $\mathrm{KCl}$ ). The chloride from $\mathrm{KCl}$ is also useful for testing because it has been shown to exacerbate dielectric failure in EWOD. ${ }^{(36)}$ For parallel-plate current leakage tests, droplets were sandwiched between the plates with a gap of $70 \mu \mathrm{m}$. For single-plate actuation tests, sessile droplets were used to measure contact angle changes and demonstrate droplet transportation.

EWOD voltage actuation was supplied by a sourcemeter (Keithley 2425) and leakage current was measured with an electrometer (Keithley 6514). Both Keithley instruments were connected to a computer with a GPIB controller (GPIB-USB-HS, National Instruments) for control and data recording. For cycling tests, mechanical relays (G5LE, OMRON) and photoMOS relays (AQW610EH PhotoMos relay, Panasonic) controlled through a DAQ (NI USB-6255, National Instruments) were used to switch electrical connections to the plates.

Current leakage was measured for both breakdown and lifetime tests. In breakdown tests, currents were measured with respect to the applied electric field. In lifetime tests, current leakage was compared for three types of biases: forward bias, reverse bias, and alternating bias. For all of the biases tested in the lifetime test, $1 \mathrm{~s}$ actuation up to $35 \mathrm{~V}$ was alternated with $1 \mathrm{~s}$ of $0 \mathrm{~V}$, i.e., $0.5 \mathrm{~Hz}$, for 1000 cycles. Contact angles were measured using a contact angle measurement program with images of the droplet during EWOD actuation captured through a CCD camera (PixeLink, model PL B742U).

\section{Results and Discussion}

\subsection{Results for sessile EWOD}

The $\mathrm{Ta}_{2} \mathrm{O}_{5}$ prepared above was first characterized for its basic performance for EWOD along with two excellent dielectrics (silicon dioxide and silicon nitride), as shown in Fig. 5. In measurements of contact angle change with applied voltage [Fig. 5(a)], an increased actuation was observed with $\mathrm{Ta}_{2} \mathrm{O}_{5}$ before contact angle saturation (at $\sim 30 \mathrm{~V}$ ). The contact angle data is replotted as the electrowetting number $E w$ in Fig. 5(b) for a better comparison with other EWOD devices in the literature. The above increased actuation compared with those of silicon oxide and silicon nitride at voltages less than $30 \mathrm{~V}$ was expected because of the significantly higher dielectric constant of $\mathrm{Ta}_{2} \mathrm{O}_{5}(21-28)$ than of silicon oxide (4-6) and silicon nitride $(\sim 8) .{ }^{(20,26)}$ Beyond the saturation voltage, the current leakage of silicon oxide increased significantly compared with those of silicon nitride and $\mathrm{Ta}_{2} \mathrm{O}_{5}$ [Fig. 5(c)].

\subsection{Results for parallel-plate EWOD}

In breakdown tests shown in Fig. 6, the measured leakage currents were low for the $\mathrm{Ta}_{2} \mathrm{O}_{5}$ samples, but increased significantly for silicon oxide samples once the voltage increased beyond $5 \mathrm{~V}$ or the field beyond $30 \mathrm{MV} / \mathrm{m}$. Silicon nitride samples broke down above $70 \mathrm{~V}$ or $425 \mathrm{MV} / \mathrm{m}$. 


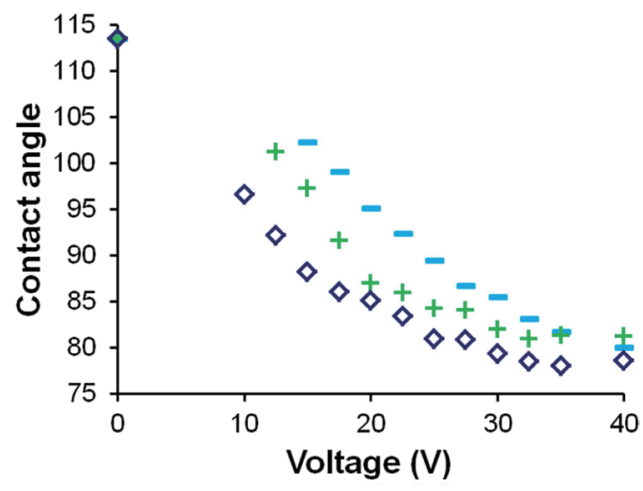

(a)

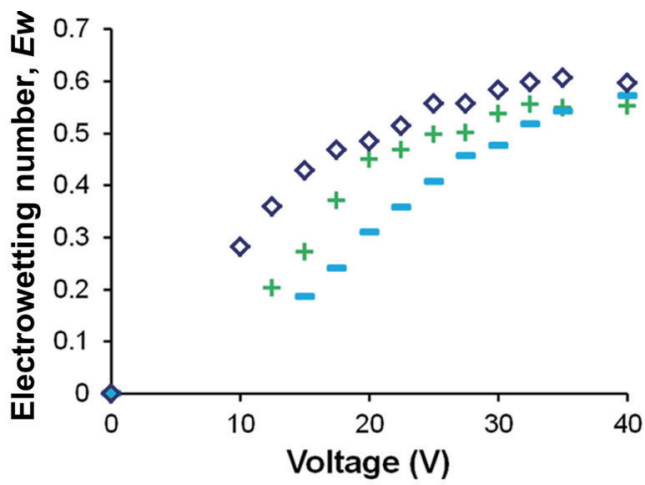

(b)

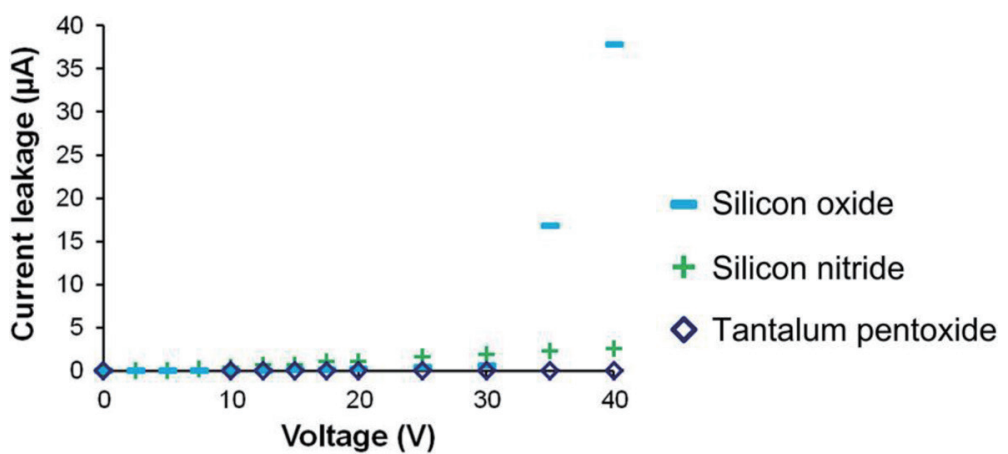

(c)

Fig. 5. (Color online) Basic performance of tantalum pentoxide as the dielectric for EWOD, evaluated with a sessile drop. (a) Contact angle, (b) electrowetting number, and (c) current leakage were measured for three different dielectrics: PECVD silicon nitride (+), PECVD silicon oxide $(-)$, and anodized tantalum pentoxide $(\diamond)$. All of the dielectrics were $165 \mathrm{~nm}$ thick. The dimensionless electrowetting number $E w$ expresses the reduction in the interfacial energy of the solid-liquid interface with the applied electric field. ${ }^{(4)}$ A larger Ew corresponds to a stronger electrowetting effect. Tantalum pentoxide shows a stronger electrowetting effect and a lower current leakage, as long as the reverse bias is maintained, corroborating previous reports. ${ }^{(27)}$

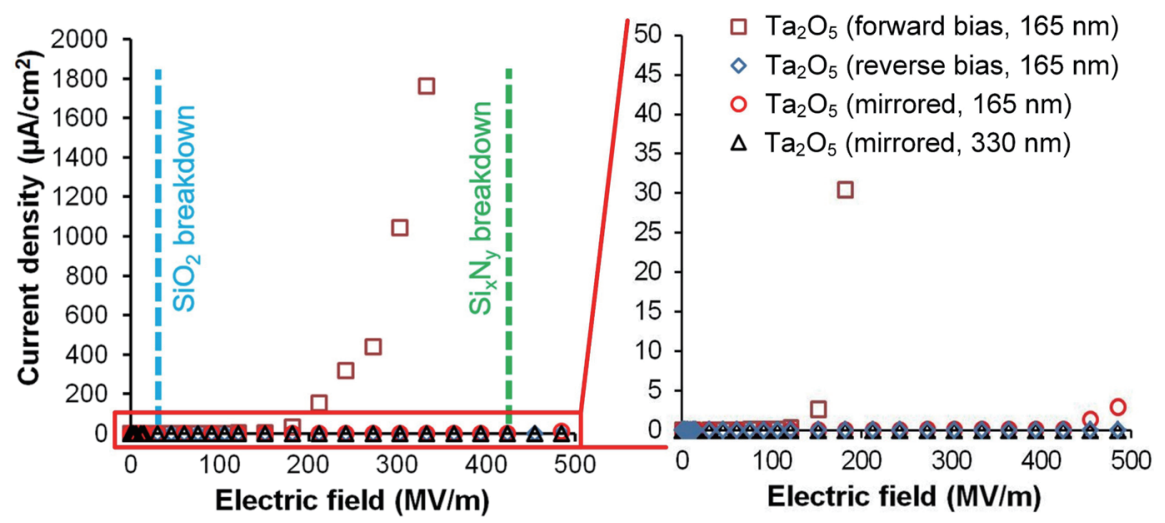

(a)

(b)

Fig. 6. (Color online) Current leakage measured as a function of electric field across tantalum pentoxide in typical (forward bias, $165 \mathrm{~nm}$; reverse bias, $165 \mathrm{~nm}$ ) and mirrored (mirrored, $165 \mathrm{~nm}$; mirrored, $330 \mathrm{~nm}$ ) EWOD devices, tested in parallel-plate configuration. (a) Breakdown voltages of PECVD $\mathrm{SiO}_{2}$ and $\mathrm{Si}_{x} \mathrm{~N}_{y}$ were measured and overlaid for comparison. (b) Zoomed in for low-leakage data. Tantalum pentoxide dielectrics in the existing configuration under reverse bias and in the mirrored configuration exhibited the lowest current leakage. 
Mirrored $\mathrm{Ta}_{2} \mathrm{O}_{5}$ samples of the same dielectric thickness broke down at a similar electric field to the silicon nitride, but with a lower current leakage. Current leakage was consistently higher for the forward-biased $\mathrm{Ta}_{2} \mathrm{O}_{5}$ than for the reverse-biased $\mathrm{Ta}_{2} \mathrm{O}_{5}$. Mirrored configuration samples were made with half the thickness $(83 \mathrm{~nm})$ of $\mathrm{Ta}_{2} \mathrm{O}_{5}$ on each plate, so that the total dielectric thickness was comparable to that on the test samples $(165 \mathrm{~nm})$ used in the typical parallel-plate configuration. In these mirrored configuration samples, the current leakage nearly matched that of the full thickness $\mathrm{Ta}_{2} \mathrm{O}_{5}$ samples under reverse bias. The lowest current leakage was measured for $\mathrm{Ta}_{2} \mathrm{O}_{5}$ in a mirrored configuration with a full $165 \mathrm{~nm}$ thickness on each plate, in which case one half of the externally applied voltage was applied to the 165 -nm-thick dielectric.

The cumulative damaging effect of forward bias actuation was clearly seen in lifetime tests with $0.5 \mathrm{~Hz}$ of $35 \mathrm{~V}$ bias voltage, as shown in Fig. 7. Under forward bias [Fig. 7(a)], the leakage current increased over repeated cycles. The leakage current did not increase steadily because the electrolysis bubbles caused the droplet to shift its position during the measurement. Under reverse bias [Fig. 7(b)], the leakage current was expected to decrease with repeated voltage cycling as a result of self-healing. ${ }^{(24,28)}$ However, the leakage current was too low for the current instrument to measure a notable decrease. For the mirrored configuration, on the other hand, no significant current change was measured. The test was repeated alternating positive and negative $35 \mathrm{~V}$ biases at $0.5 \mathrm{~Hz}$ and had similar results. For the single-plate configuration, a significant amount of current leaked during the forward-biased periods of the cycles but not during the reverse-biased periods, as expected. ${ }^{(27)}$ No significant current change was measured for the mirrored configuration.

By using anodized dielectrics in a mirrored configuration, the current leakage was limited during the actuation of a parallel-plate EWOD device, regardless of voltage bias. The fabrication of the mirrored configuration is simpler than adding a sputtering step to deposit a valve metal oxide to address the bias issue. ${ }^{(27)}$ However, its use of an equal dielectric thickness on both plates is somewhat different from that in the case of the typical parallel-plate EWOD devices, which uses the main dielectric only on the plate with patterned EWOD electrodes and leaves the other plate with a much thinner dielectric or just the hydrophobic topcoat.

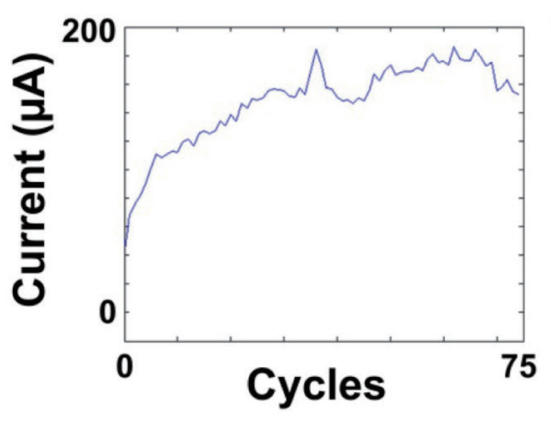

(a)

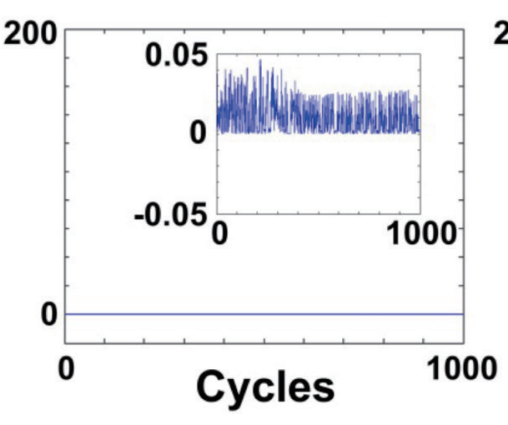

(b)

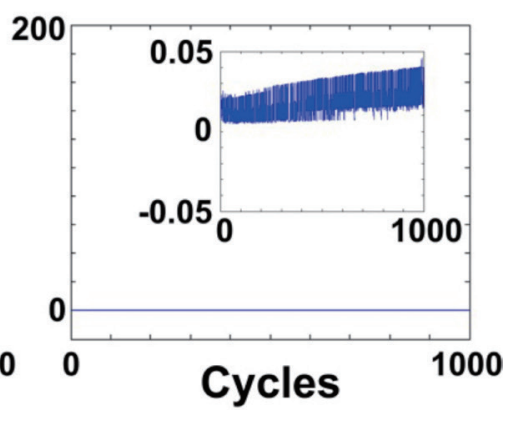

(c)

Fig. 7. (Color online) Measurement of current leakage under cycles of voltage pulse $(0.5 \mathrm{~Hz} ; 50 \%$ duty cycle) applied to a droplet on 165-nm-thick tantalum pentoxide in (a,b) typical and (c) mirrored, parallel-plate configurations. (a) The leakage current was significant for forward bias. (b) The leakage current was negligible for reverse bias. (c) For the mirrored configuration, the leakage current was negligible for both biases as well as an alternating bias. 


\subsection{Results for coplanar EWOD}

In the case of coplanar EWOD devices, where both the actuation and reference electrodes are fabricated on the same plate, ${ }^{(35)}$ a mirrored configuration was incorporated with the anodization of only a single plate. Because the valve metal oxide dielectric would still cover both the actuation and ground electrodes, its current rectifying effect should also oppose itself. In this section, the viability of the anodized dielectric in the mirrored configuration is expanded to coplanar actuation, which is the electrode configuration needed for most single-plate EWOD devices.

In sessile droplet actuation with a $5 \mu \mathrm{L}$ droplet, the current leakage was lower than $15 \mathrm{nA}$ (over a $3 \mathrm{~mm}^{2}$ area) for an approximately $20^{\circ}$ contact angle change using $25 \mathrm{~V}$, as shown in Fig. 8. In a separate coplanar design with neighboring square electrodes, a $2.5 \mu \mathrm{L}$ droplet was moved across the electrodes, as shown in Fig. 9, using an actuation voltage of $25 \mathrm{~V}$ with an average leakage current of $55 \mathrm{nA}$ during the actuation. The current leakage was somewhat higher for the coplanar EWOD than for the parallel-plate EWOD, considering that 165 -nm-thick $\mathrm{Ta}_{2} \mathrm{O}_{5}$ covered both the actuation and ground electrodes as in the parallel-plate mirrored configuration

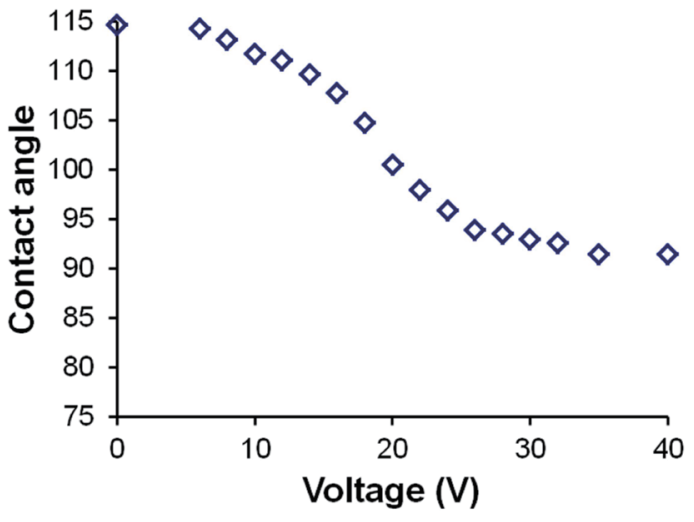

(a)

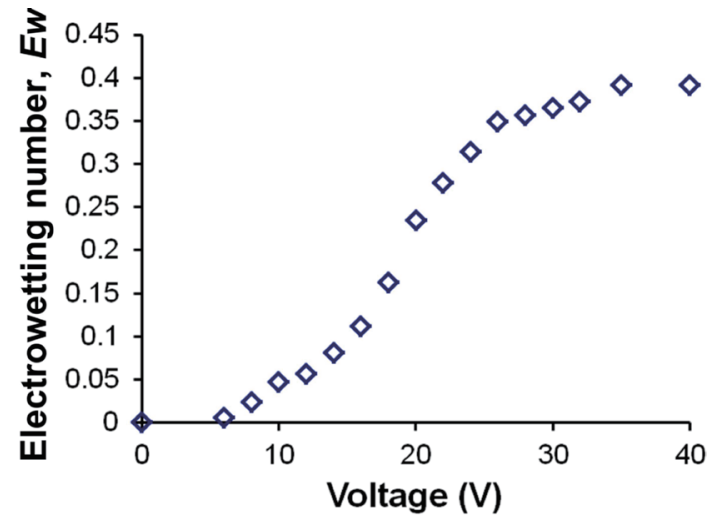

(b)

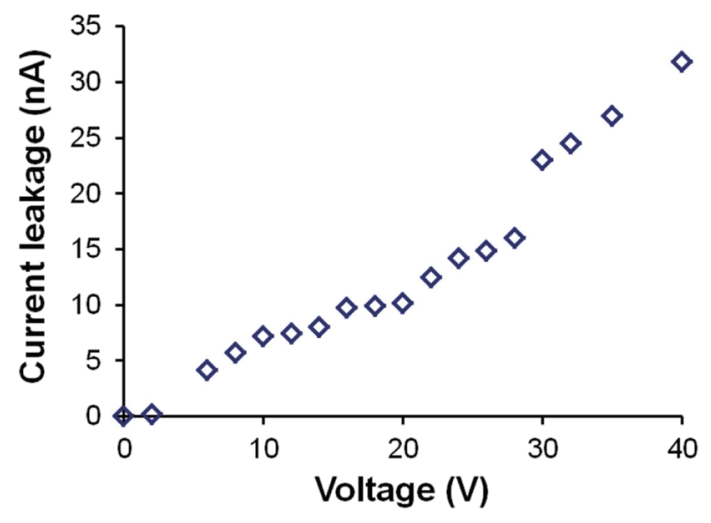

(c)

Fig. 8. (Color online) Basic performance for coplanar EWOD. (a) Contact angle, (b) electrowetting number, and (c) current leakage during EWOD actuation measured on coplanar EWOD electrodes with 165-nm-thick tantalum pentoxide of mirror configuration as the dielectric. Note the unit for current leakage is nA, not $\mu \mathrm{A}$. 


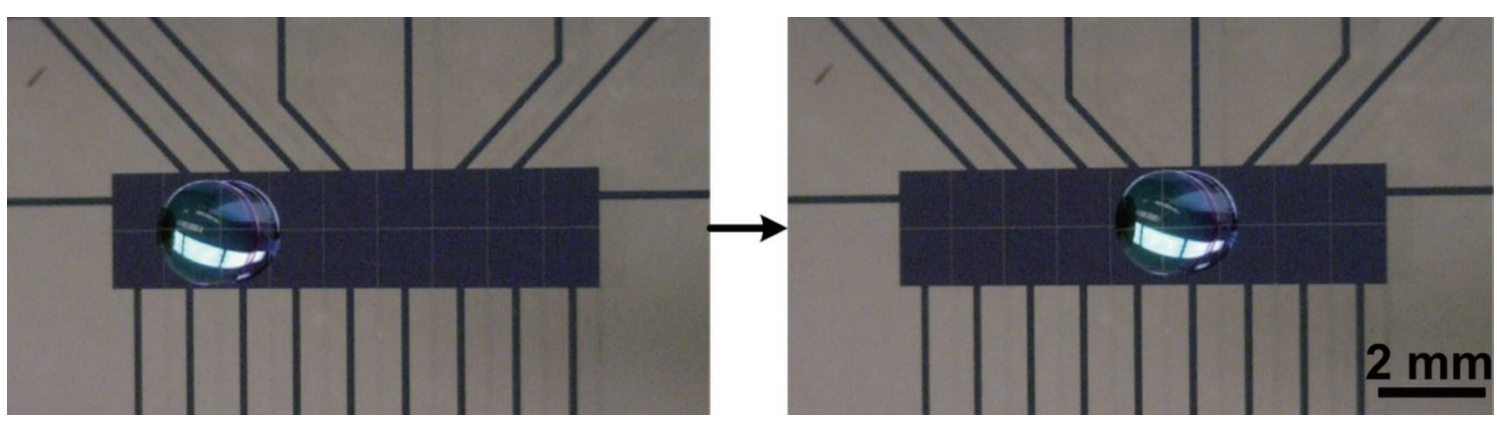

Fig. 9. (Color online) Transportation of $2.5 \mu \mathrm{L}$ droplet on a coplanar, open EWOD device with 165-nm-thick tantalum pentoxide dielectric in mirror configuration, using $25 \mathrm{~V}_{\mathrm{DC}}$ actuation signals.

with full thickness on both plates. The higher leakage for the coplanar EWOD may be due to an incomplete insulation along the side edges of tantalum electrodes during the anodization as the electric field for anodized growth was applied perpendicularly to the surface, while EWOD actuation involves an electric field parallel to the surface applied along gaps between activated and grounded electrodes. In such a case, growing more $\mathrm{Ta}_{2} \mathrm{O}_{5}$ by an additional anodization step using neighboring electrodes as counter electrodes can improve sidewall insulation.

\section{Conclusions}

The mirrored configuration for valve metal oxide dielectrics was proposed and verified using $\mathrm{Ta}_{2} \mathrm{O}_{5}$ in both parallel-plate and coplanar EWOD devices. In lifetime tests, the mirrored configuration had negligible current leakage over 1000 actuation cycles. In breakdown tests, the mirrored configuration did not suffer from the polarity effect, unlike the existing $\mathrm{Ta}_{2} \mathrm{O}_{5}$ EWOD devices. In comparison with PECVD silicon nitride and silicon oxide, the current leakage was lower across the $\mathrm{Ta}_{2} \mathrm{O}_{5}$ dielectrics. Droplet movement was demonstrated on an open EWOD plate, which has coplanar electrodes covered with $\mathrm{Ta}_{2} \mathrm{O}_{5}$, while exhibiting little current leakage as expected from the mirrored configuration.

In this study, we confirmed that valve metal oxides are a viable dielectric material for EWOD regardless of the bias applied in a mirrored configuration. A simple $\mathrm{Ta}_{2} \mathrm{O}_{5}$ anodization process was used for testing, but the dielectric performance may be improved with further process optimization. Because the conduction mechanism through metal oxides depends on the conditions in which the oxide is formed and the processing both before and after oxide growth, ${ }^{(26)}$ additional treatments, such as doping and thermal annealing, ${ }^{(37,38)}$ may be taken to improve the dielectric properties.

\section{Acknowledgments}

This work was supported by the Department of Energy (Grant DE-SC0005056), the National Science Foundation (Grants 1711708 and 1720499), the Volgenau Endowed Chair in Engineering 
at UCLA, and the UCLA Foundation from a donation made by Ralph \& Marjorie Crump for the UCLA Crump Institute for Molecular Imaging. We thank Lian-Xin Huang, R. Michael van Dam, and Jia Li for helpful discussions and Hoc Ngo for Ta sputtering.

\section{References}

1 S. Hackwood and G. Beni: Appl. Phys. Lett. 38 (1981) 207.

2 M. G. Pollack, R. B. Fair, and A. D. Shenderov: Appl. Phys. Lett. 77 (2000) 1725.

3 J. Lee, H. Moon, J. Fowler, T. Schoellhammer, and C.-J. Kim: Sens. Actuators, A 95 (2002) 259. https://doi. org/10.1016/S0924-4247(01)00734-8

4 W. C. Nelson and C.-J. Kim: J. Adhes. Sci. Technol. 26 (2012) 1747. https://doi.org/10.1163/156856111X599562

5 B. Berge: Comptes rendus de l'Académie des sciences, Série II 317 (1993) 157.

6 S. K. Cho, Y. Zhao, and C.-J. Kim: Lab Chip 7 (2007) 490. https://doi.org/10.1039/b615665g

7 B. Berge and J. Peseux: Eur. Phys. J. E 3 (2000) 159.

8 G. Cha, C.-J. Kim, and Y. S. Ju: App1. Therm. Eng. 98 (2016) 189. https://doi.org/10.1016/ j.applthermaleng.2015.11.098

9 P. Sen and C.-J. Kim: J. Microelectromech. Syst. 18 (2009) 174. https://doi.org/10.1109/JMEMS.2008.2008624

10 T. Krupenkin and J. A. Taylor: Nat. Commun. 2 (2011) 448.

11 Banpurkar, M. Duits, D. van den Ende, and F. Mugele: Langmuir 25 (2009) 1245.

12 W. C. Nelson, H. P. Kavehpour, and C.-J. Kim: Lab Chip 14 (2011) 2424. https://doi.org/10.1039/c01c00691b

13 M. J. Jebrail, M. S. Bartsch, and K. D. Patel: Lab Chip 12 (2012) 2452.

14 M. J. Jebrail, N. Assem, J. M. Mudrik, M. D. Dryden, K. Lin, A. K. Yudin, and A. R. Wheeler: J. Flow Chem. 2 (2012) 103.

15 E. Kirby and A. R. Wheeler: Anal. Chem. 85 (2013) 6178.

16 H. Moon, A. R. Wheeler, R. L. Garrell, J. A. Loo, and C.-J. Kim: Lab Chip 6 (2006) 1213. https://doi. org/10.1039/b601954d

17 L. Zhu, Y. Feng, X. Ye, J. Feng, Y. Wu, and Z. Zhou: J. Adhes. Sci. Technol. 26 (2012) 2113.

18 S.-K. Fan, P.-W. Huang, T.-T. Wang, and Y.-H. Peng: Lab Chip 8 (2008) 1325.

19 H. Moon, S. K. Cho, R. L. Garrell, and C.-J. Kim: J. Appl. Phys. 92 (2002) 4080. https://doi. org/10.1063/1.1504171

20 H. Liu, S. Dharmatilleke, D. K. Maurya, and A. A. O. Tay: Microsyst. Technol. 16 (2010) 449.

21 R. Renaudot, V. Agache, Y. Fouillet, M. Kumemura, L. Jalabert, D. Collard, and H. Fujita: Microfluid. Nanofluid. 15 (2013) 297.

22 Nishino: J. Power Sources 60 (1996) 137.

23 Y. Li, W. Parkes, L. I. Haworth, and A. Ross: J. Microelectromech. Syst. 16 (2008) 1481.

24 M. Dhindsa, J. Heikenfeld, W. Weekamp, and S. Kuiper: Langmuir 27 (2011) 5665. https://doi.org/10.1021/ la1051468

25 M. Mibus, C. Jensen, X. Hu, C. Knospe, M. L. Reed, and G. Zangari: J. Appl. Phys. 114 (2013) 014901.

26 M. Lohrengel: Mater. Sci. Eng. R. Rep. 11 (1993) 243.

27 L.-X. Huang, B. Koo, and C.-J. Kim: J. Microelectromech. Syst. 22 (2013) 253. https://doi.org/10.1109/ JMEMS.2012.2233719

28 V. Fraioli: IRE Trans. Compon. Parts 5 (1958) 72.

29 N. B. Crane, A. A. Volinsky, P. Mishra, A. Rajgadkar, and M. Khodayari: Appl. Phys. Lett. 96 (2010) 104103.

30 W. Nelson, C. M. Lynch, and N. B. Crane: Lab Chip 11 (2011) 2149.

31 R. W. Berry and D. J. Sloan: Proc. IRE 47 (1959) 1070.

32 S. Chen and C.-J. Kim: Proc. IEEE Int. Conf. MEMS (2014) 1011. https://doi.org/10.1109/ MEMSYS.2014.6765815

33 J. D. Sloppy, D. D. Macdonald, and E. C. Dickey: J. Electrochem. Soc. 157 (2010) C157.

34 L. Young: J. Electrochem. Soc. 124 (1997) 528.

35 U.-C. Yi and C.-J. Kim: J. Micromech. Microeng. 16 (2006) 2053. https://doi.org/10.1088/0960-1317/16/10/018

36 Raj, M. Dhindsa, N. R. Smith, R. Laughlin, and J. Heikenfeld: Langmuir 25 (2009) 12387.

37 T. B. Tripp and K. B. Foley: J. Electrochem. Soc. 137 (1990) 2528.

38 L. Pulfrey, P. Wilcox, and L. Young: J. Appl. Phys. 40 (1969) 3891. 